\title{
Rapid host expansion of an introduced parasite, the spiny rat louse Polyplax spinulosa (Psocodea: Phthiraptera: Polyplacidae), among endemic rodents in Australia
}

Wei Wang ${ }^{1}$, Lance A. Durden² and Renfu Shao ${ }^{{ }^{*}}$

\begin{abstract}
Background: Historical European exploration and colonization resulted in the introduction of four species of rodents to the Australian continent from Eurasia: the brown rat, Rattus norvegicus, the black rat, R. rattus, the Pacific rat, R. exulans, and the house mouse, Mus musculus. The spread of these rodents created opportunities for their co-introduced sucking lice to parasitize and adapt to endemic rodents in Australia.

Methods: We collected sucking lice from rodent specimens in seven museums across Australia. We identified the spiny rat louse, Polyplax spinulosa, based on morphology. We sequenced the mitochondrial cox 1 and $r r n L$ genes of $P$. spinulosa specimens and constructed a phylogenetic tree with $\mathrm{rrnL}$ sequences.

Results: We examined 989 rodent specimens of 54 species and collected 2111 adult sucking lice and 1064 nymphal sucking lice. We found that $P$. spinulosa had nearly doubled its host range by parasitizing at least six endemic rodent species in Australia. The other two introduced lice, P. serrata and Hoplopleura pacifica, however, have apparently failed to expand to any endemic rodents in Australia. Our analysis of mitochondrial rrn L gene sequences divided P. spinulosa into two genotypes (European vs Southeast Asian), which differ by 7.5\%; both genotypes were introduced into Australia and then expanded their host ranges to include endemic rodents.

Conclusions: The earliest record of a European ship landing in Australia was in 1606, followed by British settlement in 1788. The expansion of $P$. spinulosa to at least six endemic rodent species in Australia has therefore occurred in the time frame of 200 to 400 years, which is extremely rapid relative to its host expansion to eight native rat species in Eurasia in $\sim 16$ million years since it diverged from $P$. serrata. The host expansion of $P$. spinulosa is remarkable for a blood-sucking louse and is in stark contrast to the absence of host expansion by P. serrata and H. pacifica. Comparison among these three introduced sucking lice indicated that both louse-specific factors and host-specific factors can contribute to the success or failure of host expansion.
\end{abstract}

Keywords: Sucking lice, Rodents, Host-parasite relationships, Invasive species, Psocodea, Phthiraptera, Polyplacidae, Polyplax spinulosa

*Correspondence: rshao@usc.edu.au

${ }^{1}$ GeneCology Research Centre, School of Science and Engineering, University of the Sunshine Coast, Maroochydore, QLD 4556, Australia

Full list of author information is available at the end of the article

(c) The Author(s) 2020. This article is licensed under a Creative Commons Attribution 4.0 International License, which permits use, sharing, adaptation, distribution and reproduction in any medium or format, as long as you give appropriate credit to the original author(s) and the source, provide a link to the Creative Commons licence, and indicate if changes were made. The images or other third party material in this article are included in the article's Creative Commons licence, unless indicated otherwise in a credit line to the material. If material is not included in the article's Creative Commons licence and your intended use is not permitted by statutory regulation or exceeds the permitted use, you will need to obtain permission directly from the copyright holder. To view a copy of this licence, visit http://creativeco mmons.org/licenses/by/4.0/. The Creative Commons Public Domain Dedication waiver (http://creativecommons.org/publicdomain/ zero/1.0/) applies to the data made available in this article, unless otherwise stated in a credit line to the data. 


\section{Background}

Blood-sucking lice (Psocodea: Phthiraptera: Anoplura) are wingless obligate permanent ectoparasites of eutherian mammals, with piercing mouthparts and dorsoventrally flattened, almost oval-shaped bodies [1, 2]. Several species of sucking lice are known as disease vectors, transferring pathogens to hosts and causing louse-borne diseases [3-6]. Heavy infestation of sucking lice can also cause host hypersensitivity, dermatitis and even anemia [3, 6]. More than 540 species of sucking lice, which are all in the suborder Anoplura, have been described from 840 species of eutherian mammals [7]. Sucking lice are highly host-specific in comparison to other ectoparasites such as some chewing lice and most mites, ticks and fleas [3, 8]. Most sucking louse species parasitize a single host species or even a specific region of the host body, e.g. in humans, head lice are found only on head hair, body lice on clothes and pubic lice mainly on pubic hair [7, 9]. A small proportion of sucking louse species parasitize two or more host species [7], e.g. the spiny rat louse, Polyplax spinulosa (Burmeister, 1839) (Polyplacidae), has been recorded from nine species of rats: black rat, Rattus rattus (Linnaeus, 1758) (Asia), brown rat, Rattus norvegicus (Berkenhout, 1796) (Eurasia), greater bandicoot rat, Bandicota indica (Bechstein, 1800) (Asia), Asian rat, Rattus tanezumi (Temminck, 1844) (Asia), Himalayan field rat, Rattus nitidus (Hodgson, 1845) (Asia), Turkestan rat, Rattus pyctoris (Hodgson, 1845) (Asia), Polynesian rat, Rattus exulans (Peale, 1848) (Southeast Asia), long-haired rat, Rattus villosissimus (Waite, 1898) (Australia), and rice field rat, Rattus argentiventer (Robinson \& Kloss, 1916) (Southeast Asia) [7, 10].

With over 2000 extant species in 33 families, the order Rodentia is the most diversified mammalian order [11]. Within the Rodentia, the family Muridae is highly speciose, with 730 currently recognized species in 150 genera distributed in Eurasia, Africa and Australia [11]. Sixtythree native rodent species (including extinct species) have been recorded in Australia; all of them are in the subfamily Murinae [11-13]. Australian native rodents originated in southern Asia and colonized Australia in two main migration events, known as the old endemics and the new endemics, respectively [12]. The old endemic murines began to colonize Australia at the end of the Miocene period, 5-8 million years ago (MYA); the new endemics arrived in Australia around 1-2 MYA [11-13]. In Australia, the old endemics have diverged into 13 genera with 56 species [11-13] and colonized a wide range of terrestrial environments including arid areas, forests, wet open woodlands, swamps, waterways and grasslands from tropical to middle latitudes and from sea level to mountain peaks [13]. The new endemics have diverged into seven species, all in the genus Rattus: bush rat, R. fuscipes (Waterhouse, 1839), swamp rat, $R$. lutreolus (Gray, 1841), cane field rat, $R$. sordidus (Gould, 1858), Cape York rat, R. leucopus (Gray, 1867), long-haired rat, R. villosissimus (Waite, 1898), dusky rat, $R$. colletti (Thomas, 1904) and pale field rat, $R$. tunneyi (Thomas, 1904). Three of the seven new endemic species inhabit forests (e.g. rainforest, coastal forest and eucalypt forest); the others occur in open grasslands or arid environments [13-15] (Fig. 1). The seven native Rattus species in Australia are divided into two phylogenetic groups: the Australian group with six species and the New Guinean group with only $R$. leucopus [16].

Thirteen species of blood-sucking lice have been recorded from 12 species of endemic rodents in Australia [10, 17-21]. All of the 13 species are in the genus Hoplopleura (Hoplopleuridae) except for P. spinulosa, which was introduced into Australia with the brown rat, $R$. norvegicus, and the black rat, $R$. rattus [22]. Wang et al. [10] reported recently that $P$. spinulosa has switched to the endemic $R$. villosissimus in Australia. In this study, we investigated further the host expansion of $P$. spinulosa and two other introduced sucking lice, Polyplax serrata (Burmeister, 1839) and Hoplopleura pacifica Ewing, 1924, among endemic rodents in Australia. We show that $P$. spinulosa has expanded its host range to at least six endemic rodent species (including $R$. villosissimus) in Australia; P. serrata and H. pacifica however, have not expanded to any endemic rodents. The host expansion of P. spinulosa in Australia has occurred within a short time frame of 200 to 400 years, which is remarkably rapid relative to its host expansion in Eurasia where the species originated.

\section{Methods}

Collection of sucking lice and morphological examination Sucking lice were collected from ethanol-preserved rodent specimens in seven museums across Australia (Table 1). Lice were dislodged from the host pelage with a head louse comb using a modified "cocktail shaking" technique [21]. First, rodents were put on a tray, one at a time; the pelage was gently and thoroughly combed with a head louse comb. Then, the rodent was transferred into a jar, which was filled with $80 \%$ ethanol, capped and gently shaken for $\sim 1$ min to dislodge lice from the host pelage. The rodent was then removed from the jar. Finally, the ethanol solution was filtered through a fine mesh and the filtrate was examined under a dissecting microscope (Nikon SMZ800N, Tokyo, Japan) for lice. Any lice present were removed from the mesh, kept in labelled individual vials (one per host specimen), filled with ethanol and stored at $-20{ }^{\circ} \mathrm{C}$. For morphological examination, 


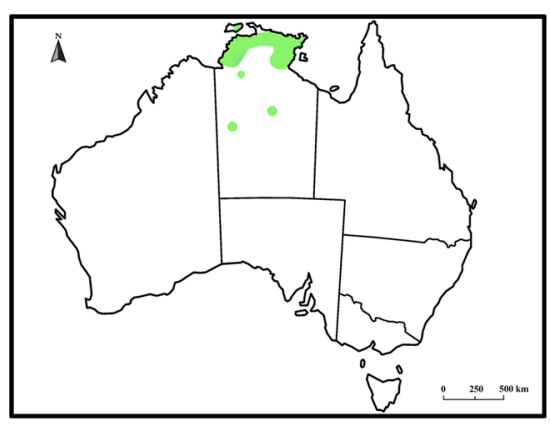

Rattus colletti

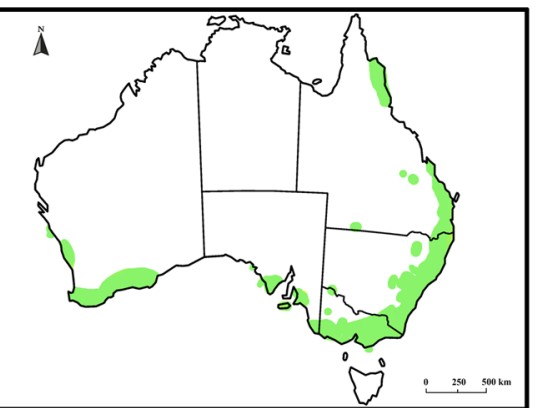

Rattus fuscipes

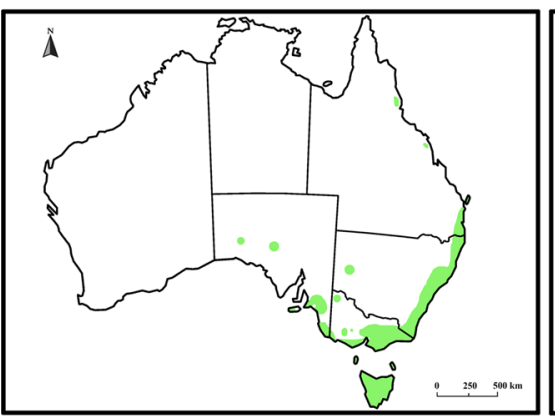

Rattus lutreolus

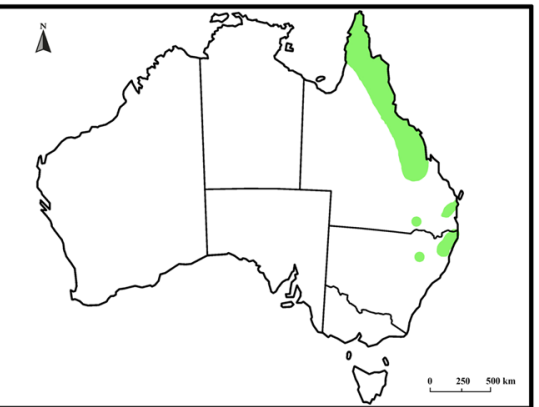

Rattus sordidus

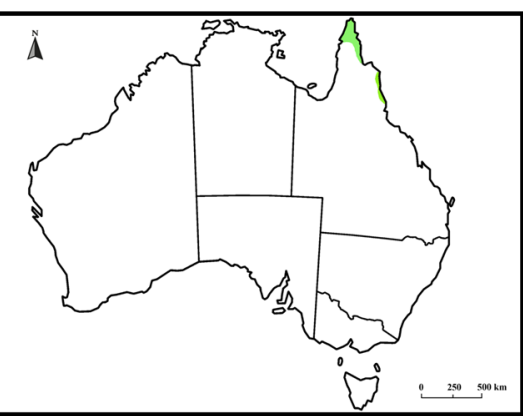

Rattus leucopus

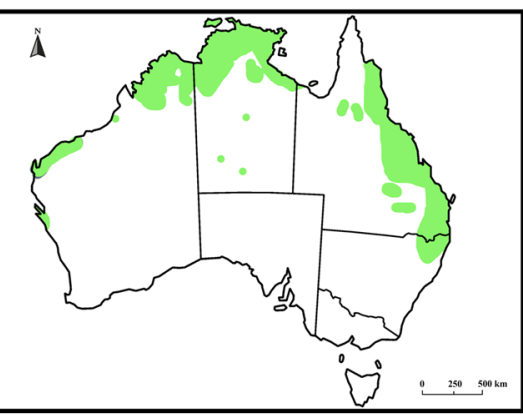

Rattus tunneyi

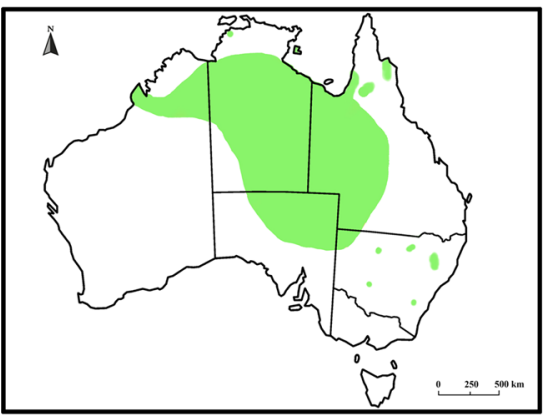

Rattus villosissimus

Fig. 1 Geographical distribution of Rattus colletti, Rattus fuscipes, Rattus leucopus, Rattus /utreolus, Rattus sordidus, Rattus tunneyi and Rattus villosissimus in Australia (adapted from https://ala.org.au)

louse specimens were mounted on microscope slides in Canada balsam: intact specimens with minimal gut contents were selected and cleared in $\mathrm{KOH}(20 \%)$ and then sequentially immersed in acetic acid (10\%), acid fuchsin (1\%), ethanol (40\%, 70\% and 100\%), pure clove oil and finally mounted on slides [23]. Louse specimens were examined and measured with a photomicroscope (Nikon ECLIPSE Ts 2, Tokyo, Japan). The following distinct features were examined for the identification of $P$. spinulosa: the 3rd segment of antenna of the male and female; the shape of the thoracic sternal plate and the shape and setation of the paratergal plates. Descriptive format and abbreviations follow Kim et al. [24].

\section{DNA extraction, amplification and sequence analysis}

Total DNA was extracted from individual louse specimens with DNeasy Tissue and Blood Kit (Qiagen, Hilden, Germany), following manufacturer protocols. We extracted DNA using a non-grinding method [25, 26] (Table 2). After DNA extraction, louse exoskeletons were mounted on microscope slides and examined morphologically. A fragment of the mitochondrial cox 1 gene ( $\sim 600 \mathrm{bp})$ was amplified by polymerase chain reaction (PCR) with primers mtd6 (5'-GGA GGA TTT GGA AAT TGA TTA GTT CC-3') and mtd11 (5'-ACT GTA AAT ATA TGA TGA GCT CA-3') [27]. A fragment of the mitochondrial $r r n L$ gene $(\sim 320 \mathrm{bp})$ was amplified with 
Table 1 Collections of sucking lice from murine rodent specimens in seven museums across Australia

\begin{tabular}{|c|c|c|c|c|c|c|c|c|c|c|c|c|c|}
\hline \multirow[t]{2}{*}{ Rodent species $(n=54)$} & \multicolumn{7}{|c|}{ No. of rodents sampled $(n=989)$} & \multirow[t]{2}{*}{$\begin{array}{l}\text { Rodents with } \\
\text { sucking lice }\end{array}$} & \multirow[t]{2}{*}{$\begin{array}{l}\text { Rodents with } \\
\text { Polyplax lice }\end{array}$} & \multicolumn{2}{|c|}{$\begin{array}{l}\text { No. of all sucking } \\
\text { lice collected }\end{array}$} & \multicolumn{2}{|c|}{$\begin{array}{l}\text { No. of Polyplax } \\
\text { spinulosa } \\
\text { collected }\end{array}$} \\
\hline & QM & MV & AM & WAM & SA & NT & QVM & & & Adults & Nymphs & Adults & Nymphs \\
\hline Conilurus penicillatus & - & - & - & 4 & 7 & 11 & - & 6 & - & 24 & 50 & - & - \\
\hline Hydromys chrysogaster & - & - & 6 & - & - & - & 5 & 3 & - & 24 & 11 & - & - \\
\hline Leggadina forresti & 20 & 25 & - & - & - & - & - & 16 & 1 & 37 & 104 & 1 & - \\
\hline Leggadina lakedownensis & 13 & - & - & 10 & - & 13 & - & 7 & - & 44 & 26 & - & - \\
\hline Leporillus conditor & - & 1 & - & - & 3 & 1 & - & 3 & - & 29 & 16 & - & - \\
\hline Mastacomys fuscus & - & - & 2 & - & - & - & - & 2 & - & 74 & 30 & - & - \\
\hline Melomys burtoni & 1 & - & - & - & - & 5 & - & 4 & - & 9 & 7 & - & - \\
\hline Melomys capensis & - & - & - & - & 3 & - & - & - & - & 20 & 13 & - & - \\
\hline Melomys cervinipes & 25 & - & - & - & - & - & - & 22 & - & 121 & - & - & - \\
\hline Mesembriomys gouldi & 3 & 6 & - & - & - & - & - & 3 & - & 30 & 34 & - & - \\
\hline Mesembriomys macrurus & - & 1 & - & 12 & 4 & 3 & - & 3 & 1 & 11 & 7 & 1 & - \\
\hline Notomys alexis & - & 29 & 7 & - & - & - & - & 8 & - & 11 & 7 & - & - \\
\hline Notomys aquilo & - & - & 1 & - & - & 6 & - & - & - & - & - & - & - \\
\hline Notomys cervinus & - & 6 & 4 & - & 10 & - & - & 5 & - & 32 & 18 & - & - \\
\hline Notomys fuscus & - & 21 & - & - & 11 & - & - & 8 & - & 13 & 16 & - & - \\
\hline Notomys longicaudatus ${ }^{\text {a }}$ & - & 22 & - & - & 1 & - & - & 6 & - & 6 & 6 & - & - \\
\hline Notomys mitchelli & - & 20 & - & 13 & 7 & - & - & 6 & - & 16 & 22 & - & - \\
\hline Pseudomys albocinereus & - & - & - & 30 & - & - & - & 3 & - & 18 & 39 & - & - \\
\hline Pseudomys apodemoides & - & - & - & - & 19 & - & - & 10 & - & 26 & 7 & - & - \\
\hline Pseudomys australis & - & 27 & - & - & - & - & - & 14 & - & 36 & 11 & - & - \\
\hline Pseudomys bolami & - & - & 11 & - & - & - & - & 3 & - & 13 & 1 & - & - \\
\hline Pseudomys calabyi & - & - & - & 9 & - & 18 & - & 1 & - & - & 1 & - & - \\
\hline Pseudomys chapmani & - & - & - & 20 & 2 & - & - & 2 & - & 1 & 2 & - & - \\
\hline Pseudomys delicatulus & 28 & - & 14 & - & - & - & - & 9 & - & 16 & 12 & - & - \\
\hline Pseudomys desertor & 5 & - & - & - & - & - & - & 4 & - & 20 & 35 & - & - \\
\hline Pseudomys fieldi & - & - & - & 20 & 3 & - & - & - & - & - & - & - & - \\
\hline Pseudomys gracilicaudatus & 13 & - & 5 & - & - & - & - & 7 & - & 11 & 28 & - & - \\
\hline Pseudomys hermannsburgensis & 2 & - & - & 9 & - & - & - & 6 & - & 29 & 7 & - & - \\
\hline Pseudomys higginsi & - & - & - & - & - & - & 6 & 4 & - & 4 & 7 & - & - \\
\hline Pseudomys laborifex & - & - & - & 14 & - & 25 & - & 4 & - & 5 & 6 & - & - \\
\hline Pseudomys nanus & - & - & - & - & - & 12 & - & 5 & - & 66 & 32 & - & - \\
\hline Pseudomys novaehollandiae & 5 & - & 19 & - & - & - & 4 & 8 & - & 17 & 6 & - & - \\
\hline Pseudomys occidentalis & - & - & - & 14 & - & - & - & 3 & 1 & 119 & 29 & 47 & 15 \\
\hline Pseudomys patrius & 19 & - & - & - & - & - & - & 1 & - & 1 & - & - & - \\
\hline Pseudomys shortridgei & - & - & - & 11 & 4 & - & - & - & - & - & - & - & - \\
\hline Uromys caudimaculatus & - & - & 3 & - & - & - & - & - & - & - & - & - & - \\
\hline Uromys hadrourus & 1 & - & - & - & - & - & - & 1 & - & 2 & 4 & - & - \\
\hline Xeromys myoides & 14 & - & - & - & - & 1 & - & 3 & - & 2 & 3 & - & - \\
\hline Zyzomys argurus & - & - & - & - & - & 9 & - & 3 & - & 9 & 5 & - & - \\
\hline Zyzomys maini & - & - & - & - & - & 3 & - & - & - & - & - & - & - \\
\hline Zyzomys palatalis & - & - & - & - & - & 9 & - & 3 & - & 12 & 15 & - & - \\
\hline Zyzomys pedunculatus & - & 11 & - & - & 1 & - & - & 4 & - & 3 & 7 & - & - \\
\hline Zyzomys woodwardi & - & - & - & 11 & 2 & - & - & 1 & - & - & 1 & - & - \\
\hline Rattus colletti & 9 & - & - & - & - & 13 & - & 7 & 1 & 38 & 79 & 1 & - \\
\hline Rattus fuscipes & 37 & - & 2 & - & - & - & - & 21 & 6 & 75 & 28 & 53 & 20 \\
\hline Rattus leucopus & 27 & - & 6 & 5 & - & - & - & 16 & - & 23 & 51 & - & - \\
\hline Rattus lutreolus & 8 & - & 5 & - & - & - & 7 & 12 & 4 & 52 & 23 & 12 & 13 \\
\hline
\end{tabular}


Table 1 (continued)

\begin{tabular}{|c|c|c|c|c|c|c|c|c|c|c|c|c|c|}
\hline \multirow[t]{2}{*}{ Rodent species $(n=54)$} & \multicolumn{7}{|c|}{ No. of rodents sampled $(n=989)$} & \multirow[t]{2}{*}{$\begin{array}{l}\text { Rodents with } \\
\text { sucking lice }\end{array}$} & \multirow[t]{2}{*}{$\begin{array}{l}\text { Rodents with } \\
\text { Polyplax lice }\end{array}$} & \multicolumn{2}{|c|}{$\begin{array}{l}\text { No. of all sucking } \\
\text { lice collected }\end{array}$} & \multicolumn{2}{|c|}{$\begin{array}{l}\text { No. of Polyplax } \\
\text { spinulosa } \\
\text { collected }\end{array}$} \\
\hline & QM & MV & AM & WAM & SA & NT & QVM & & & Adults & Nymphs & Adults & Nymphs \\
\hline Rattus sordidus & 15 & - & 6 & - & - & - & - & 18 & 10 & 52 & 15 & 24 & 9 \\
\hline Rattus tunneyi & 24 & - & - & - & - & 3 & - & 27 & 15 & 99 & 60 & 39 & 23 \\
\hline Rattus villosissimus & 27 & - & - & - & - & - & - & 27 & 17 & 826 & 109 & 706 & 45 \\
\hline Rattus rattus $^{\mathrm{b}}$ & 2 & 7 & - & - & - & - & - & 5 & 5 & 35 & 73 & 35 & 73 \\
\hline Mus musculus ${ }^{b}$ & 1 & - & - & - & - & 6 & - & 1 & - & - & 1 & - & - \\
\hline Rattus exulans ${ }^{\mathrm{b}}$ & - & 1 & - & - & - & - & - & 0 & - & - & - & - & - \\
\hline Rattus norvegicus ${ }^{\mathrm{b}}$ & - & - & - & - & - & 3 & - & 0 & - & - & - & - & - \\
\hline Total & 299 & 177 & 91 & 182 & 77 & 141 & 22 & 337 & 60 & 2111 & 1064 & 919 & 198 \\
\hline
\end{tabular}

a Extinct species

b Introduced species to Australia

Abbreviations: QM, Queensland Museum; MV, Museums Victoria; AM, Australian Museum; WAM, Western Australian Museum; NTM, Museum and Art Gallery of the Northern Territory; QVM, Queen Victoria Museum and Art Gallery; SA, South Australian Museum

Note: "-" indicates no specimens were checked or collected

primers 16SF (5'-TTA ATT CAA CAT CGA GGT CGC AA- $\left.3^{\prime}\right)$ and Lx16SR (5'-GAC TGT GCT AAG GTA GCA TAA T-3') [28]. PCR conditions were: initial step of $1 \mathrm{~min}$ at $94{ }^{\circ} \mathrm{C}$, followed by 40 cycles of $10 \mathrm{~s}$ at $98^{\circ} \mathrm{C}, 5 \mathrm{~s}$ at $40{ }^{\circ} \mathrm{C}$ (for cox 1 ) and $52{ }^{\circ} \mathrm{C}$ (for $r r n L$ ) and $5 \mathrm{~s}$ at $72{ }^{\circ} \mathrm{C}$ and a final extension for $30 \mathrm{~s}$ at $72{ }^{\circ} \mathrm{C}$. These primers target highly conserved sequence motifs among arthropods. PCR amplifications were $25 \mu \mathrm{l}$ each using $12.5 \mu \mathrm{l}$ of PrimeSTAR Max Premix Mix (Takara, Shiga, Japan), $9.5 \mu \mathrm{H}_{2} \mathrm{O}$, $1 \mu \mathrm{l}$ of each primer and $1 \mu \mathrm{l}$ of DNA template. PCR products were purified using Wizard ${ }^{\circledR}$ SV Gel Clean-Up System (Promega, Madison, USA), following manufacturer instructions. Purified cox 1 and $r r n L$ amplicons were sequenced in both directions with the Sanger method at the Australian Genome Research Facilities (AGRF) in Brisbane, Australia. Sequence reads were assembled using Geneious 11.0.2; gene identities were verified by BLAST searches of GenBank.

\section{Phylogenetic analysis of $r r n L$ gene fragments}

Multiple sequence alignments were created with Geneious 11.0.2 software [29]. An unrooted neighbor-joining (NJ) consensus tree of $r r n L$ gene fragments was constructed using the distance matrix calculated by the Kimura-Nei model of evolution as implemented in the Geneious 11.0.2 [29]. A bootstrap analysis using 1000 replicates was performed on the resulting tree for node support.

\section{Results}

\section{New hosts of Polyplax spinulosa among endemic rodents} in Australia

We examined 989 rodent specimens of 54 species (50 endemic species, four introduced species) in seven museums across Australia, and collected 2111 adult sucking lice and 1064 nymphal sucking lice (Table 1). We also examined 13 adult sucking lice and three nymphal sucking lice collected from two $R$. rattus specimens from Sabah (Borneo) and Sunshine Coast (Queensland), respectively (Table 2). We did not examine specimens of the other 13 endemic rodent species (nine of them extinct) due to the unavailability of specimens to us. Of the 2124 adult sucking lice collected, 932 specimens (i.e. 43.9\%) were identified as Polyplax spinulosa; the remaining specimens were Hoplopleura spp., some of which represent undescribed species. Polyplax spinulosa was found on 63 individual rodents, i.e. $6.4 \%$ of the total number of rodents we examined $(n=991)$; these 63 individual rodents were collected over a period of 65 years (19552019) from three old endemic species, six new endemic species and one introduced species (Table 2). The number of $P$. spinulosa we collected varied substantially among the 10 host species. A single adult P. spinulosa was collected from the old endemic species, Leggadina forresti and Mesembriomys macrurus, respectively; another single adult $P$. spinulosa was collected from the new endemic species, $R$. colletti (Table 2). Twelve to 706 adults of $P$. spinulosa were collected from each of the other six old or new endemic species, together with 9 to 73 Polyplax nymphs. Forty-eight adult specimens of $P$. spinulosa were collected from the introduced black rat, $R$. rattus, together with 76 Polyplax nymphs (Table 2). The intensity of $P$. spinulosa presence also varied substantially from one adult louse to 260 adult lice on an individual host rodent (Table 2). Of the 63 individual rodents that were parasitized by $P$. spinulosa, five rodents also hosted Hoplopleura spp., giving a double-infestation rate of $8.2 \%$; in 
Table 2 Murine rodent specimens $(n=63)$ from which Polyplax spinulosa was collected

\begin{tabular}{|c|c|c|c|c|c|c|c|}
\hline Host species & Sample ID & Storage & Locality of collection & $\begin{array}{l}\text { Polyplax spinulosa } \\
\text { collected } \\
{\left[n=932(201)^{\mathrm{a}}\right]}\end{array}$ & $\begin{array}{l}\text { Hoplopleura } \\
\text { spp. collected } \\
{\left[n=33(26)^{\mathrm{a}}\right]}\end{array}$ & $\begin{array}{l}\text { Collection } \\
\text { date }\end{array}$ & Museum or collector \\
\hline Leggadina forresti & JM4346 & Spirit & $\begin{array}{l}\text { Benditoota Waterhole, Queens- } \\
\text { land }\left(25^{\circ} 37^{\prime} S, 139^{\circ} 48^{\prime} \mathrm{E}\right)\end{array}$ & $1(0)$ & 0 & 28-Sep-1982 & $\begin{array}{l}\text { Queensland } \\
\text { Museum }\end{array}$ \\
\hline $\begin{array}{l}\text { Mesembriomys } \\
\text { macrurus }\end{array}$ & C7597 & Spirit & $\begin{array}{l}\text { Balanbrinni Ck, W Coast, Gulf } \\
\text { of Carpentaria inland from } \\
\text { Macarthur River, Northern Ter- } \\
\text { ritory }\left(16^{\circ} 58^{\prime} \mathrm{S}, 135^{\circ} 32^{\prime} \mathrm{E}\right)\end{array}$ & $1(0)$ & 0 & No record & Museums Victoria \\
\hline $\begin{array}{l}\text { Pseudomys occiden- } \\
\text { talis }\end{array}$ & M43324 & Spirit & $\begin{array}{l}\text { Bluff Knoll, Western Australia } \\
\left(34^{\circ} 22^{\prime} 00^{\prime \prime} \mathrm{S}, 118^{\circ} 15^{\prime} 00^{\prime \prime} \mathrm{E}\right)\end{array}$ & $47(15)$ & 0 & 28-Sep-1994 & $\begin{array}{l}\text { Western Australian } \\
\text { Museum }\end{array}$ \\
\hline Rattus colletti & $J 21881$ & Spirit & Northern Territory & $1(0)$ & 0 & No record & $\begin{array}{l}\text { Queensland } \\
\text { Museum }\end{array}$ \\
\hline \multirow[t]{6}{*}{ Rattus fuscipes } & $J 2722$ & Spirit & $\begin{array}{l}\text { Brisbane, Fortitude Valley, } \\
\text { Queensland }\left(27^{\circ} 28^{\prime} \mathrm{S}, 153^{\circ} 2^{\prime} \mathrm{E}\right)\end{array}$ & $1(3)$ & 0 & No record & $\begin{array}{l}\text { Queensland } \\
\text { Museum }\end{array}$ \\
\hline & $J 2769$ & Spirit & $\begin{array}{l}\text { Brisbane, Fortitude Valley, } \\
\text { Queensland }\left(27^{\circ} 28^{\prime} \mathrm{S}, 153^{\circ} 2^{\prime} \mathrm{E}\right)\end{array}$ & $1(0)$ & 0 & No record & $\begin{array}{l}\text { Queensland } \\
\text { Museum }\end{array}$ \\
\hline & $J 20068$ & Spirit & $\begin{array}{l}\text { Gallangowan, Queensland } \\
\left(26^{\circ} 26^{\prime} \mathrm{S}, 152^{\circ} 20^{\prime} \mathrm{E}\right)\end{array}$ & $1(1)$ & 0 & 1-Aug-1955 & $\begin{array}{l}\text { Queensland } \\
\text { Museum }\end{array}$ \\
\hline & $J 20084$ & Spirit & $\begin{array}{l}\text { Euramoo Ck, Danbulla, NE } \\
\text { Atherton, Queensland }\left(17^{\circ} 9^{\prime} \mathrm{S} \text {, }\right. \\
\left.145^{\circ} 37^{\prime} \mathrm{E}\right)\end{array}$ & $1(1)$ & 0 & 26-Aug-1956 & $\begin{array}{l}\text { Queensland } \\
\text { Museum }\end{array}$ \\
\hline & $J 20113$ & Spirit & $\begin{array}{l}\text { Walsh Camp, } 11.2 \mathrm{~km} \text { SW Ather- } \\
\text { ton, Queensland }\left(17^{\circ} 20^{\prime} \mathrm{S},\right. \\
\left.145^{\circ} 25^{\prime} \mathrm{E}\right)\end{array}$ & $46(15)$ & 0 & 8-Aug-1956 & $\begin{array}{l}\text { Queensland } \\
\text { Museum }\end{array}$ \\
\hline & M31441 & Spirit & $\begin{array}{l}\text { Comerong Is, Nowra, New } \\
\text { South Wales }\left(34^{\circ} 53^{\prime} 37^{\prime \prime} \mathrm{S},\right. \\
\left.150^{\circ} 44^{\prime} 56^{\prime \prime} \mathrm{E}\right)\end{array}$ & $3(0)$ & 0 & 25-Jan-1995 & Australian Museum \\
\hline \multirow[t]{4}{*}{ Rattus /utreolus } & $R L A^{b, c, d}$ & Frozen & $\begin{array}{l}\text { Chuwar Ipswich, Queens- } \\
\text { land }\left(27^{\circ} 22^{\prime} 53.71^{\prime \prime} \mathrm{S},\right. \\
\left.152^{\circ} 47^{\prime} 12.72^{\prime \prime} \mathrm{E}\right)\end{array}$ & $4(3)$ & 0 & No record & $\begin{array}{l}\text { Queensland } \\
\text { Museum }\end{array}$ \\
\hline & $J M 12492$ & Spirit & $\begin{array}{l}\text { Camira, Queensland }\left(27^{\circ} 37^{\prime} \mathrm{S},\right. \\
\left.152^{\circ} 56^{\prime} \mathrm{E}\right)\end{array}$ & $2(0)$ & 0 & 12-May-1998 & $\begin{array}{l}\text { Queensland } \\
\text { Museum }\end{array}$ \\
\hline & $J M 12711$ & Spirit & $\begin{array}{l}\text { Tinaroo Dam, Queensland } \\
\left(17^{\circ} 10^{\prime} S, 145^{\circ} 33^{\prime} \mathrm{E}\right)\end{array}$ & $4(10)$ & 0 & 20-Feb-1963 & $\begin{array}{l}\text { Queensland } \\
\text { Museum }\end{array}$ \\
\hline & JM12771 & Spirit & $\begin{array}{l}\text { Boonah Shire, Mt Barney NP, } \\
\text { Cronan Ck, Queensland } \\
\left(28^{\circ} 18^{\prime} 20^{\prime \prime} \mathrm{S}, 152^{\circ} 41^{\prime} 25^{\prime \prime} \mathrm{E}\right)\end{array}$ & $2(0)$ & 0 & 5-Oct-1993 & $\begin{array}{l}\text { Queensland } \\
\text { Museum }\end{array}$ \\
\hline \multirow[t]{7}{*}{ Rattus rattus } & $Z 65055^{b, c, d}$ & Spirit & $\begin{array}{l}\text { Victoria Range Road, } 1.1 \text { km } \\
\text { WNW }\left(296.7^{\circ}\right) \text { of intersection } \\
\text { of Victoria Range Road and } \\
\text { Sawmill Track, Grampians } \\
\text { National Park, Victoria }\end{array}$ & $9(15)$ & 0 & Nov-2017 & Museums Victoria \\
\hline & $R S 92^{b, c, e}$ & Spirit & Tuaran, Sabah (Borneo) & $9(0)$ & 0 & 1-Mar-2008 & Konstans Wells \\
\hline & C28523 & Spirit & $\begin{array}{l}\text { Yarra Valley Metropolitan Park, } \\
\text { Victoria }\end{array}$ & $22(57)$ & 0 & 26-Jul-1990 & Museums Victoria \\
\hline & C36839 & Spirit & 12 Leura Ave, Rosanna, Victoria & $1(0)$ & 0 & 20-Jun-2011 & Museums Victoria \\
\hline & C37126 & Spirit & $\begin{array}{l}\text { Cooinda Burrong Scout Camp, } \\
\text { Grampians National Park, } \\
\text { Victoria }\end{array}$ & $2(0)$ & 0 & 22-Nov-2012 & Museums Victoria \\
\hline & Z65054 & Spirit & $\begin{array}{l}\text { Victoria Range Road, } 1.1 \mathrm{~km} \\
\text { WNW }\left(296.7^{\circ}\right) \text { of intersection } \\
\text { of Victoria Range Road and } \\
\text { Sawmill Track, Grampians } \\
\text { National Park, Victoria }\end{array}$ & $1(1)$ & 0 & Nov-2017 & Museums Victoria \\
\hline & $R S 361^{b, c, d}$ & Fresh & $\begin{array}{l}\text { Australian Zoo Wildlife Hospital, } \\
\text { Queensland }\end{array}$ & $4(3)$ & 0 & 9-Aug-2019 & $\begin{array}{l}\text { Renfu Shao, Yalun } \\
\text { Dong }\end{array}$ \\
\hline Rattus sordidus & M34647 & Spirit & Queensland & $1(1)$ & 0 & 2-Mar-2000 & Australian Museum \\
\hline
\end{tabular}


Table 2 (continued)

\begin{tabular}{|c|c|c|c|c|c|c|c|}
\hline Host species & Sample ID & Storage & Locality of collection & $\begin{array}{l}\text { Polyplax spinulosa } \\
\text { collected } \\
{\left[n=932(201)^{a}\right]}\end{array}$ & $\begin{array}{l}\text { Hoplopleura } \\
\text { spp. collected } \\
{\left[n=33(26)^{a}\right]}\end{array}$ & $\begin{array}{l}\text { Collection } \\
\text { date }\end{array}$ & Museum or collector \\
\hline & $J 20061$ & Spirit & $\begin{array}{l}\text { Walsh Camp, } 11.2 \mathrm{~km} \text { SW Ather- } \\
\text { ton, Queensland }\left(17^{\circ} 20^{\prime} \mathrm{S}\right. \\
\left.145^{\circ} 25^{\prime} \mathrm{E}\right)\end{array}$ & $4(2)$ & 0 & 13-Aug-1956 & $\begin{array}{l}\text { Queensland } \\
\text { Museum }\end{array}$ \\
\hline & $J 20062$ & Spirit & $\begin{array}{l}\text { Walsh Camp, } 11.2 \mathrm{~km} \text { SW Ather- } \\
\text { ton, Queensland }\left(17^{\circ} 20^{\prime} \mathrm{S}\right. \\
\left.145^{\circ} 25^{\prime} \mathrm{E}\right)\end{array}$ & $1(0)$ & 0 & 8-Aug-1956 & $\begin{array}{l}\text { Queensland } \\
\text { Museum }\end{array}$ \\
\hline & JM4093 & Spirit & $\begin{array}{l}\text { York Downs, Queensland } \\
\left(12^{\circ} 45^{\prime} 11^{\prime \prime} \mathrm{S}, 142^{\circ} 18^{\prime} 36^{\prime \prime} \mathrm{E}\right)\end{array}$ & $1(0)$ & 0 & 14-May-1981 & $\begin{array}{l}\text { Queensland } \\
\text { Museum }\end{array}$ \\
\hline & JM13985 & Spirit & $\begin{array}{l}\text { Janie Ck Mth, } 12 \mathrm{~km} \text { SW of Cul- } \\
\text { len Point, N of Weipa, Queens- } \\
\text { land }\left(12^{\circ} 2^{\prime} 13^{\prime \prime} \mathrm{S}, 141^{\circ} 49^{\prime} 26^{\prime \prime} \mathrm{E}\right)\end{array}$ & $1(0)$ & 0 & 9-Sep-1981 & $\begin{array}{l}\text { Queensland } \\
\text { Museum }\end{array}$ \\
\hline & JM13986 & Spirit & $\begin{array}{l}\text { Paperbark Flats, NW of Weipa, } \\
\text { Queensland }\left(12^{\circ} 6^{\prime} 33^{\prime \prime} \mathrm{S},\right. \\
\left.142^{\circ} 21^{\prime} 52^{\prime \prime} \mathrm{E}\right)\end{array}$ & $1(0)$ & 0 & 7-Sep-1981 & $\begin{array}{l}\text { Queensland } \\
\text { Museum }\end{array}$ \\
\hline & JM15192 & Spirit & $\begin{array}{l}\text { Innisfail, Queensland } \\
\left(17^{\circ} 32^{\prime} 55^{\prime \prime} \mathrm{S}, 145^{\circ} 51^{\prime} 02^{\prime \prime} \mathrm{E}\right)\end{array}$ & $0(1)$ & 0 & 10-Sep-2002 & $\begin{array}{l}\text { Queensland } \\
\text { Museum }\end{array}$ \\
\hline & JM15199 & Spirit & $\begin{array}{l}\text { Innisfail, Queensland } \\
\quad\left(17^{\circ} 32^{\prime} 55^{\prime \prime} \mathrm{S}, 145^{\circ} 51^{\prime} 02^{\prime \prime} \mathrm{E}\right)\end{array}$ & $12(2)$ & 0 & 10-Sep-2002 & $\begin{array}{l}\text { Queensland } \\
\text { Museum }\end{array}$ \\
\hline & $J M 23200$ & Spirit & Queensland & $2(3)$ & 0 & No record & $\begin{array}{l}\text { Queensland } \\
\text { Museum }\end{array}$ \\
\hline & JM23201 & Spirit & Queensland & $1(0)$ & 0 & No record & $\begin{array}{l}\text { Queensland } \\
\text { Museum }\end{array}$ \\
\hline \multirow[t]{13}{*}{ Rattus tunneyi } & $N 19187^{b, c}$ & Frozen & $\begin{array}{l}\text { Mockers RD, Fernvale, Queens- } \\
\text { land }\left(27^{\circ} 29^{\prime} \mathrm{S}, 152^{\circ} 40^{\prime} \mathrm{E}\right)\end{array}$ & $10(0)$ & 0 & 21-Feb-2010 & $\begin{array}{l}\text { Queensland } \\
\text { Museum }\end{array}$ \\
\hline & JM8136 & Spirit & $\begin{array}{l}\text { Leynora Downs, } 25 \mathrm{~km} \mathrm{~S} \\
\text { of Rolleston, Queensland } \\
\left(24^{\circ} 38^{\prime} \mathrm{S}, 148^{\circ} 50^{\prime} \mathrm{E}\right)\end{array}$ & $1(0)$ & 0 & 24-Aug-1990 & $\begin{array}{l}\text { Queensland } \\
\text { Museum }\end{array}$ \\
\hline & JM12645 & Spirit & $\begin{array}{l}\text { Sunrise Hstd, NW Injune, } \\
\text { Queensland }\left(25^{\circ} 20^{\prime} 6^{\prime \prime} S \text {, }\right. \\
\left.148^{\circ} 5^{\prime} 47^{\prime \prime} \mathrm{E}\right)\end{array}$ & $1(0)$ & 0 & 7-Oct-1996 & $\begin{array}{l}\text { Queensland } \\
\text { Museum }\end{array}$ \\
\hline & JM13324 & Spirit & $\begin{array}{l}\text { Maryborough, } 2 \mathrm{~km} \text { from } \\
\text { Maryborough \& Hervey Bay } \\
\text { Rd, Queensland }\left(25^{\circ} 32^{\prime} \mathrm{S},\right. \\
\left.152^{\circ} 42^{\prime} \mathrm{E}\right)\end{array}$ & $1(0)$ & 0 & 18-Aug-1999 & $\begin{array}{l}\text { Queensland } \\
\text { Museum }\end{array}$ \\
\hline & JM13785 & Spirit & $\begin{array}{l}\text { Northern Downs District, } 15 \\
\text { km E Dalby, Queensland } \\
\left(27^{\circ} 9^{\prime} 24^{\prime \prime} \mathrm{S}, 151^{\circ} 27^{\prime} 50^{\prime \prime} \mathrm{E}\right)\end{array}$ & $0(3)$ & 0 & 18-Mar-1999 & $\begin{array}{l}\text { Queensland } \\
\text { Museum }\end{array}$ \\
\hline & JM13786 & Spirit & $\begin{array}{l}\text { Northern Downs District, } 15 \\
\text { km E Dalby, Queensland } \\
\left(27^{\circ} 1^{\prime} 44^{\prime \prime} S, 151^{\circ} 15^{\prime} 59^{\prime \prime} E\right)\end{array}$ & $1(0)$ & 0 & 18-Mar-1999 & $\begin{array}{l}\text { Queensland } \\
\text { Museum }\end{array}$ \\
\hline & JM13788 & Spirit & $\begin{array}{l}\text { Northern Downs District, } 15 \\
\text { km E Dalby, Queensland } \\
\left(27^{\circ} 1^{\prime} 44^{\prime \prime} S, 151^{\circ} 15^{\prime} 59^{\prime \prime} E\right)\end{array}$ & $2(0)$ & 0 & 18-Mar-1999 & $\begin{array}{l}\text { Queensland } \\
\text { Museum }\end{array}$ \\
\hline & J9201 & Skin & $\begin{array}{l}\text { Gallangowan, Queensland } \\
\left(26^{\circ} 26^{\prime} \mathrm{S}, 152^{\circ} 20^{\prime} \mathrm{E}\right)\end{array}$ & $2(0)$ & 0 & No record & $\begin{array}{l}\text { Queensland } \\
\text { Museum }\end{array}$ \\
\hline & JM1333 & Spirit & $\begin{array}{l}\text { Kilcoy area, Queensland } \\
\left(26^{\circ} 57^{\prime} \mathrm{S}, 152^{\circ} 34^{\prime} \mathrm{E}\right)\end{array}$ & $1(8)$ & $1(0)$ & 7-Nov-1973 & $\begin{array}{l}\text { Queensland } \\
\text { Museum }\end{array}$ \\
\hline & JM4102 & Skin & $\begin{array}{l}\text { Red Beach, } 8 \mathrm{~km} \text { S Cullen } \\
\text { Point, Queensland }\left(12^{\circ} 1^{\prime} 5^{\prime \prime} \mathrm{S} \text {, }\right. \\
\left.141^{\circ} 53^{\prime} 55^{\prime \prime} \mathrm{E}\right)\end{array}$ & $1(0)$ & $1(0)$ & 8-Sep-1980 & $\begin{array}{l}\text { Queensland } \\
\text { Museum }\end{array}$ \\
\hline & JM6923 & Spirit & $\begin{array}{l}\text { Cecil Plains, Brisbane, Queens- } \\
\text { land }\left(27^{\circ} 32^{\prime} \mathrm{S}, 151^{\circ} 11^{\prime} \mathrm{E}\right)\end{array}$ & $3(4)$ & 0 & 1989 & $\begin{array}{l}\text { Queensland } \\
\text { Museum }\end{array}$ \\
\hline & JM7265 & Spirit & $\begin{array}{l}\text { Blue Lagoon, Moreton Is, W side, } \\
\text { Queensland }\left(27^{\circ} 6^{\prime} \mathrm{S}, 153^{\circ} 26^{\prime} \mathrm{E}\right)\end{array}$ & $1(1)$ & 0 & 6-Apr-1973 & $\begin{array}{l}\text { Queensland } \\
\text { Museum }\end{array}$ \\
\hline & J9204 & Skin & $\begin{array}{l}\text { Gallangowan, Queensland } \\
\left(26^{\circ} 26^{\prime} \mathrm{S}, 152^{\circ} 20^{\prime} \mathrm{E}\right)\end{array}$ & $12(3)$ & 0 & No record & $\begin{array}{l}\text { Queensland } \\
\text { Museum }\end{array}$ \\
\hline
\end{tabular}


Table 2 (continued)

\begin{tabular}{|c|c|c|c|c|c|c|c|}
\hline Host species & Sample ID & Storage & Locality of collection & $\begin{array}{l}\text { Polyplax spinulosa } \\
\text { collected } \\
{\left[n=932(201)^{\mathrm{a}}\right]}\end{array}$ & $\begin{array}{l}\text { Hoplopleura } \\
\text { spp. collected } \\
{\left[n=33(26)^{\mathrm{a}}\right]}\end{array}$ & $\begin{array}{l}\text { Collection } \\
\text { date }\end{array}$ & Museum or collector \\
\hline & $J 21294$ & Skin & $\begin{array}{l}\text { Archookoora, via Kingaroy, } \\
\text { Queensland }\left(26^{\circ} 44^{\prime} \mathrm{S},\right. \\
\left.151^{\circ} 48^{\prime} \mathrm{E}\right)\end{array}$ & $0(1)$ & 0 & Oct-1969 & $\begin{array}{l}\text { Queensland } \\
\text { Museum }\end{array}$ \\
\hline & $J 22596$ & Skin & $\begin{array}{l}\text { Brookfield, Gold Ck Rd, Bris- } \\
\text { bane, Queensland }\left(27^{\circ} 30^{\prime} \mathrm{S} \text {, }\right. \\
\left.152^{\circ} 55^{\prime} \mathrm{E}\right)\end{array}$ & $3(3)$ & 0 & 26-May-1972 & $\begin{array}{l}\text { Queensland } \\
\text { Museum }\end{array}$ \\
\hline \multirow[t]{17}{*}{ Rattus villosissimus } & RVA & Frozen & $\begin{array}{l}\text { Crossroads on Tonkoro Road, } \\
\text { Queensland ( } 24^{\circ} 08^{\prime} 56.6^{\prime \prime} \mathrm{S}, \\
\left.143^{\circ} 35^{\prime} 01.6^{\prime \prime} \mathrm{E}\right)\end{array}$ & $30(0)$ & 0 & 9-Mar-2011 & $\begin{array}{l}\text { Queensland } \\
\text { Museum }\end{array}$ \\
\hline & $R \vee B^{b, c, d}$ & Frozen & $\begin{array}{l}\text { Noonbah Station, Homestead, } \\
\text { Queensland }\left(24^{\circ} 06^{\prime} 27^{\prime \prime} \mathrm{S},\right. \\
\left.143^{\circ} 11^{\prime} 10^{\prime \prime} \mathrm{E}\right)\end{array}$ & $260(0)$ & 0 & 31-Jul-2011 & $\begin{array}{l}\text { Queensland } \\
\text { Museum }\end{array}$ \\
\hline & RVC & Frozen & $\begin{array}{l}\text { Queensland }\left(24^{\circ} 17^{\prime} 41.5^{\prime \prime} \mathrm{S},\right. \\
\left.143^{\circ} 19^{\prime} 48.1^{\prime \prime} \mathrm{E}\right)\end{array}$ & $150(0)$ & 0 & 2-Mar-2011 & $\begin{array}{l}\text { Queensland } \\
\text { Museum }\end{array}$ \\
\hline & RVD & Frozen & $\begin{array}{l}\text { Noonbah Station, Homestead, } \\
\text { Queensland }\left(24^{\circ} 06^{\prime} 27^{\prime \prime S},\right. \\
\left.143^{\circ} 11^{\prime} 10^{\prime \prime} \mathrm{E}\right)\end{array}$ & $20(0)$ & 0 & 10-Apr-2011 & $\begin{array}{l}\text { Queensland } \\
\text { Museum }\end{array}$ \\
\hline & RVE & Frozen & $\begin{array}{l}\text { Noonbah Station, Homestead, } \\
\text { Queensland }\left(24^{\circ} 06^{\prime} 27^{\prime \prime S} \text {, }\right. \\
\left.143^{\circ} 11^{\prime} 10^{\prime \prime} \mathrm{E}\right)\end{array}$ & $3(9)$ & 0 & No record & $\begin{array}{l}\text { Queensland } \\
\text { Museum }\end{array}$ \\
\hline & RVF & Frozen & West Queensland, Queensland & $1(0)$ & 0 & No record & $\begin{array}{l}\text { Queensland } \\
\text { Museum }\end{array}$ \\
\hline & $\mathrm{RVH}$ & Frozen & $\begin{array}{l}\text { Vergemont, Queensland } \\
\quad\left(24^{\circ} 06^{\prime} 26.0^{\prime \prime} \mathrm{S}, 143^{\circ} 11^{\prime} 10.8^{\prime \prime} \mathrm{E}\right)\end{array}$ & $23(12)$ & 0 & 15-Apr-2011 & $\begin{array}{l}\text { Queensland } \\
\text { Museum }\end{array}$ \\
\hline & RVI & Frozen & West Queensland, Queensland & $120(0)$ & 0 & No record & $\begin{array}{l}\text { Queensland } \\
\text { Museum }\end{array}$ \\
\hline & RVJ & Frozen & $\begin{array}{l}\text { Noonbah Station, Homestead, } \\
\text { Queensland }\left(24^{\circ} 06^{\prime} 27^{\prime \prime S},\right. \\
\left.143^{\circ} 11^{\prime} 10^{\prime \prime} \mathrm{E}\right)\end{array}$ & $0(5)$ & 0 & No record & $\begin{array}{l}\text { Queensland } \\
\text { Museum }\end{array}$ \\
\hline & RVK & Frozen & West Queensland, Queensland & $12(2)$ & 0 & 28-Apr-2011 & $\begin{array}{l}\text { Queensland } \\
\text { Museum }\end{array}$ \\
\hline & RVL & Frozen & $\begin{array}{l}1 \mathrm{~km} \text { east of Waterloo Bore } \\
\text { PD Dam, Queensland } \\
\left(24^{\circ} 09^{\prime} 58.9^{\prime \prime} \mathrm{S}, 143^{\circ} 14^{\prime} 58.8^{\prime \prime} \mathrm{E}\right)\end{array}$ & $50(0)$ & 0 & 24-Feb-2011 & $\begin{array}{l}\text { Queensland } \\
\text { Museum }\end{array}$ \\
\hline & RVM & Frozen & $\begin{array}{l}\text { Thomson River } 200 \mathrm{~m} \text { west } \\
\text { of bridge, Queensland } \\
\left(24^{\circ} 05^{\prime} 45.5^{\prime \prime} \mathrm{S}, 143^{\circ} 22^{\prime} 55.2^{\prime \prime} \mathrm{E}\right)\end{array}$ & $8(0)$ & 0 & 19-Apr-2011 & $\begin{array}{l}\text { Queensland } \\
\text { Museum }\end{array}$ \\
\hline & N68645 & Frozen & $\begin{array}{l}\text { Glenore Vena Park Rd. } \\
\text { Normanton, Queens- } \\
\text { land }\left(18^{\circ} 17^{\prime} 32.15^{\prime \prime} \mathrm{S}\right. \\
\left.141^{\circ} 12^{\prime} 41.85^{\prime \prime} \mathrm{E}\right)\end{array}$ & $1(0)$ & 0 & 11-Jun-2011 & $\begin{array}{l}\text { Queensland } \\
\text { Museum }\end{array}$ \\
\hline & JM4824 & Spirit & $\begin{array}{l}\text { Sandringham (61-22), Montara } \\
\text { Dune, Queensland ( } 23^{\circ} 56^{\prime} \mathrm{S}, \\
\left.138^{\circ} 47^{\prime} \mathrm{E}\right)\end{array}$ & 7 (3) & 0 & 28-Jul-1984 & $\begin{array}{l}\text { Queensland } \\
\text { Museum }\end{array}$ \\
\hline & JM4825 & Spirit & $\begin{array}{l}\text { Sandringham (61-22), Montara } \\
\text { Dune, Queensland ( } 23^{\circ} 56^{\prime} \mathrm{S} \text {, } \\
\left.138^{\circ} 47^{\prime} \mathrm{E}\right)\end{array}$ & $12(8)$ & $17(13)$ & 29-Jul-1984 & $\begin{array}{l}\text { Queensland } \\
\text { Museum }\end{array}$ \\
\hline & JM5234 & Spirit & $\begin{array}{l}\text { Marked Tree Waterhole, } 2 \mathrm{~km} \\
\text { North, Queensland }\left(23^{\circ} 17^{\prime} \mathrm{S},\right. \\
\left.138^{\circ} 9^{\prime} \mathrm{E}\right)\end{array}$ & $1(0)$ & $10(7)$ & 8-Aug-1985 & $\begin{array}{l}\text { Queensland } \\
\text { Museum }\end{array}$ \\
\hline & JM10742 & Spirit & $\begin{array}{l}\text { Diamantina Lakes, Queensland } \\
\left(23^{\circ} 40^{\prime} \mathrm{S}, 141^{\circ} 5^{\prime} \mathrm{E}\right)\end{array}$ & $8(6)$ & $4(6)$ & $\begin{array}{l}\text { 10-14 Aug- } \\
1981\end{array}$ & $\begin{array}{l}\text { Queensland } \\
\text { Museum }\end{array}$ \\
\hline
\end{tabular}

a No. of adult specimens outside parenthesis, number of nymphal specimens inside parenthesis

b Polyplax spinulosa specimens from which DNA extraction was successful

c Mitochondrial $r r n L$ gene sequencedww

d Mitochondrial cox 1 gene sequenced

e The only rodent specimen collected outside Australia in the present study 


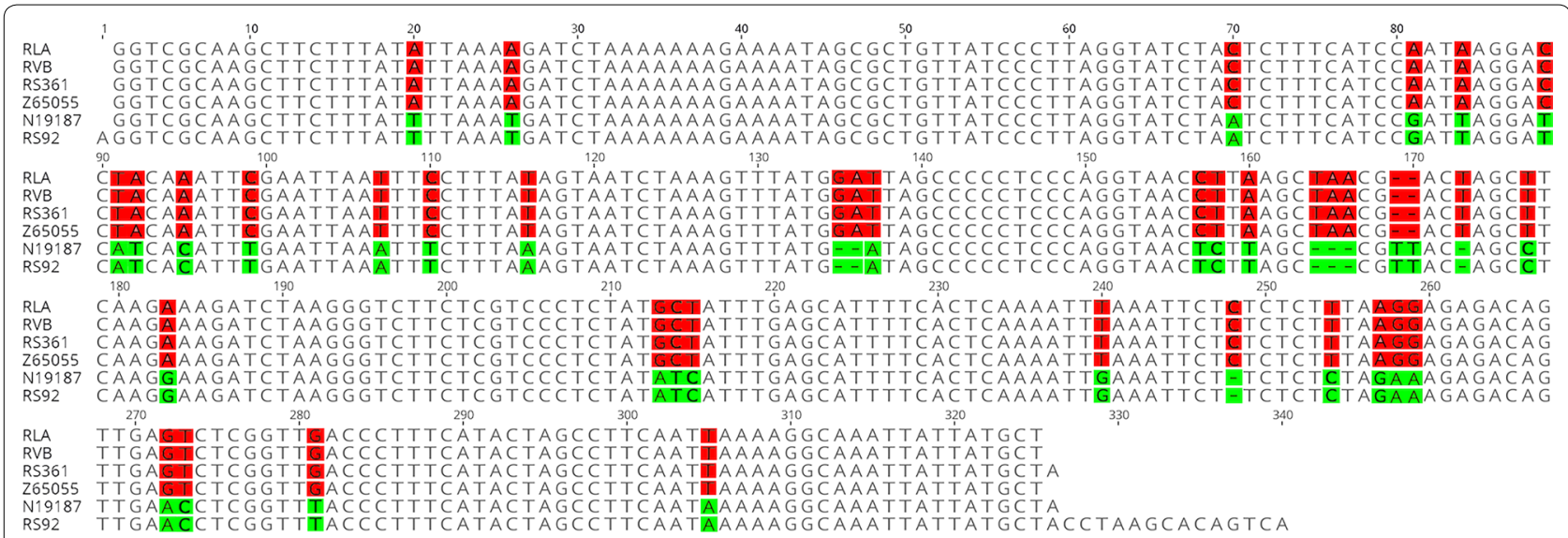

Fig. 2 Sequence alignment of the mitochondrial rrnL gene of RLA, RVB, RS361, Z65055, RS92 and N19187. Red and green shading indicates nucleotide variation between the two genotypes

each case of double-infestation, the abundance was similar between P. spinulosa and Hoplopleura spp. (Table 2).

We collected a single sucking louse from the introduced house mouse, Mus musculus ( $n=7$, Table 1), but could not identify it to either genus or species level as the specimen was an early-stage nymph. We did not find $P$. spinulosa on the other 43 endemic rodent species $(n=716)$, or on the other two introduced species: the brown rat, $R$. norvegicus $(n=3)$ and the Polynesian rat, $R$. exulans $(n=1)$. We did not find the other two species of introduced sucking lice, $P$. serrata and $H$. pacifica, on any of the 991 rodent specimens we checked (Tables 1,2).

\section{Morphology of Polyplax spinulosa recorded on endemic rodents in Australia}

To confirm the identification of $P$. spinulosa, we mounted 30 adult sucking louse specimens $\left(16 \hat{O}^{\hat{1}}, 14\right.$ \% $)$ on microscope slides and examined their morphology in detail; these 30 specimens were from 10 rodent hosts: ex $M$. macrurus (MV C7597, 19) (note: museum name abbreviation, rodent specimen registration number and number of louse specimen mounted and sex are listed hereafter), ex L. forresti (QM JM4346, 10َ), ex P. occidentalis (WAM M43324, 2犬, 1ㅇ), ex R. colletti (QM J21881, 19), ex R. fuscipes (QM J20113, 20, 19), ex R. lutreolus (MV RLA, 20, 2ᄋ), ex $R$. sordidus (QM J92310, 10, 1ㅇ), ex R. tunneyi (QM N19187, 20, 2ᄋ), ex R. villosissimus

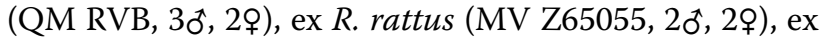
$R$. rattus (Sabah RS92, 10, 19) (note: MV for Museums Victoria, WAM for Western Australian Museum, QM for Queensland Museum) (Additional file 1: Figure S1, Additional file 2: Figure S2). We also examined all other unmounted specimens under a high-magnification binocular microscope. Polyplax spinulosa is morphologically distinct from other Polyplax species but only a few minor characters distinguish it from $P$. serrata, $P$. wallacei Durden, 1987 and P. reclinata (Nitzsch, 1864). We observed and relied on four distinct characters to identify $P$. spinulosa collected from rodent hosts. First, P. spinulosa has a shield shaped, six-sided thoracic sternal plate (Additional file 3: Figure S3), whereas $P$. reclinata has a flat anterior margin on this plate. Polyplax serrata is smaller than $P$. spinulosa in body length and its thoracic sternal plate has a rounded anterior margin [30]. The thoracic sternal plate of $P$. wallace $i$ is uniquely shield shaped with extended anterolateral angles [31]. Secondly, the spiracles of the paratergal plates of $P$. reclinata are larger than those of $P$. spinulosa. Thirdly, the setae on the paratergal plates of $P$. reclinata are longer than in $P$. spinulosa. The ventral posterior seta on the third paratergal plate of $P$. serrata is much longer than the corresponding dorsal seta, whereas in P. spinulosa both of these setae are short and about equal in length [30]. Paratergal plates VI and VII of $P$. wallacei each have two long apical setae; the dorsal posterior seta on paratergal plates I to III is longer than the corresponding ventral seta [31]. Fourth, the posterior setae on paratergal plate IV are the same length or longer than paratergal plate IV in $P$. serrata, while in P. spinulosa, the posterior setae of each paratergal plate are shorter than each corresponding paratergal plate [32, 33]. Additionally, P. reclinata parasitizes shrews in Africa and Eurasia [7] and would not be expected to parasitize murine rodents or to occur in Australia.

\section{Two genotypes of Polyplax spinulosa revealed by mitochondrial $r r n L$ gene sequences}

The vast majority of the $P$. spinulosa specimens we collected in museums were old; their hosts had been fixed in formalin prior to preservation in ethanol. Therefore, most lice collected from these hosts were not suitable 


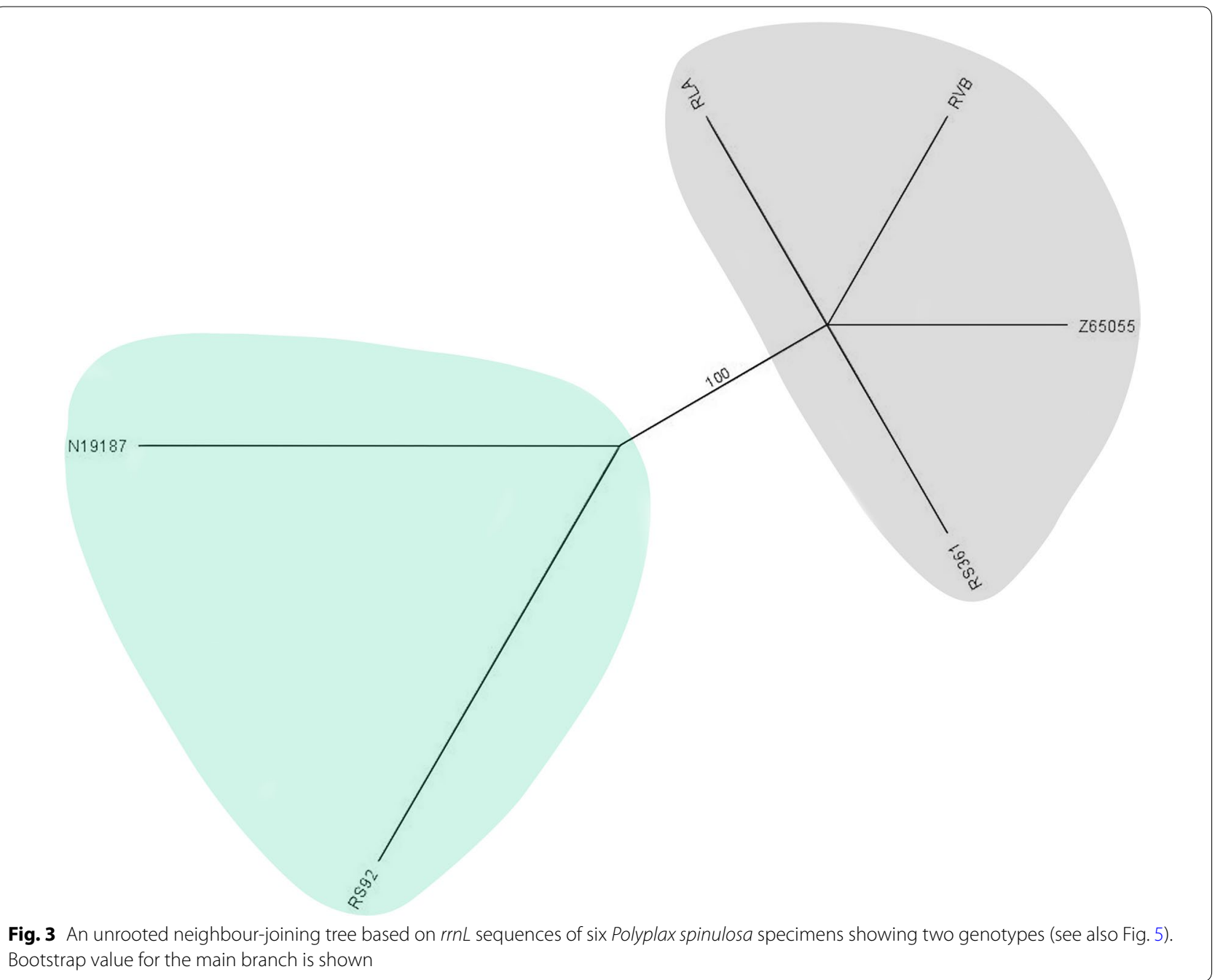

for molecular analysis. Nevertheless, we sequenced successfully the mitochondrial $r r n L$ gene fragment $(\sim 320$ bp) of six $P$. spinulosa specimens and the mitochondrial cox1 gene fragment ( $600 \mathrm{bp})$ of four $P$. spinulosa specimens (Table 2). Comparison of the $r r n L$ sequences revealed two genotypes that differed by $7.5 \%$ (Figs. 2, $3)$. Genotype 1 was shared ( $100 \%$ identical) by four $P$. spinulosa specimens found on different Rattus species in Queensland and Victoria: R. lutreolus (QM RLA), $R$. villosissimus (QM RVB) and $R$. rattus (MV Z65055 and RS361 from Queensland), respectively. We also obtained the $\operatorname{cox} 1$ sequences of these four specimens, which had $>99.3 \%$ identity to each other and to the published cox 1 sequence of $P$. spinulosa collected from $R$. norvegicus in the Czech Republic (GenBank: EU162140 [34]) (Additional file 4: Figure S4), indicating a European origin of Genotype 1. The Genotype $2 \mathrm{rrnL}$ sequence was shared (100\% identical) by two P. spinulosa specimens found on R. tunneyi from Queensland (QM N19187) and R. rattus
(RS92) from Sabah (Borneo) (Table 2, Fig. 2), thus indicating a Southeast Asian origin of this genotype. We were unable to obtain a cox1 sequence from these two $P$. spinulosa specimens (QM N19187 and RS92) despite repeated attempts. Although we did not have data on the hosts, the two genotypes of $P$. spinulosa are likely related to the two forms of $R$. rattus in Australia: the Oceanic form that came with the First Fleet and the Asian form [35].

\section{Discussion}

Host expansion of Polyplax spinulosa has been extremely rapid in Australia compared to Eurasia

The old endemic rodents arrived on the Australian continent 5-8 MYA at the end of the Miocene period; the new endemics arrived around 1-2 MYA [11-13]. Hoplopleura appears to be the only genus of sucking lice carried into Australia by the old endemic rodents because all of the known sucking louse species that parasitize endemic rodents are in this genus except $P$. spinulosa [10]. The 

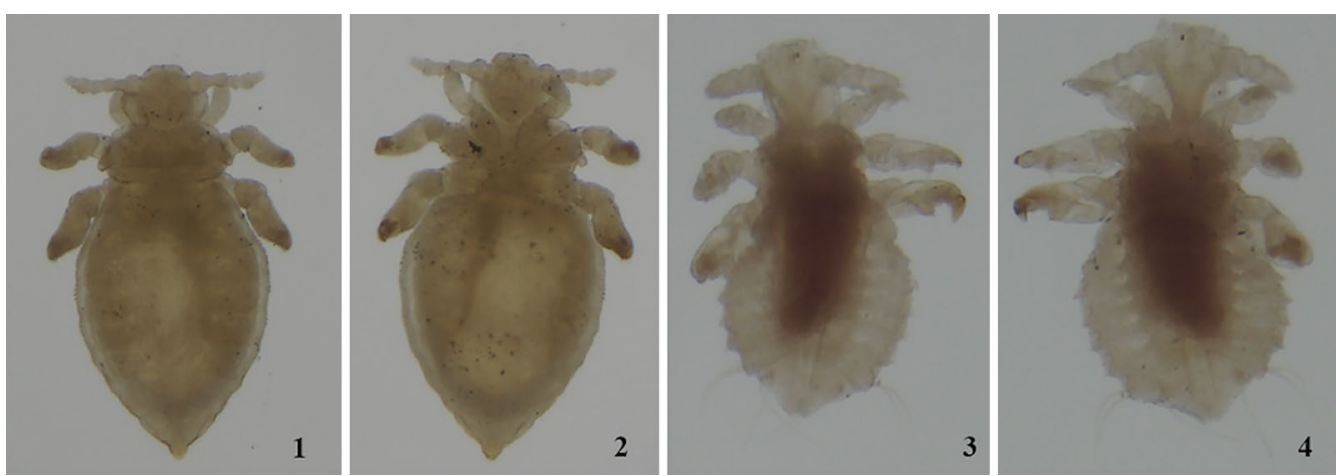

Fig. 4 Uncleared nymphs of Hoplopleura sp. from Notomys mitchelli: (1) dorsal surface and (2) ventral surface. Uncleared nymphs of Polyplax spinulosa: (3) dorsal surface and (4) ventral surface

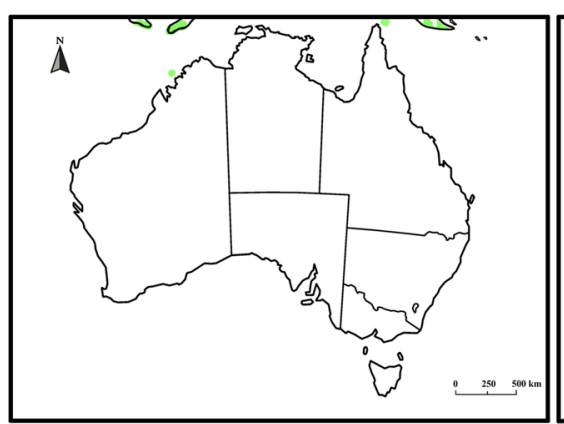

Rattus exulans

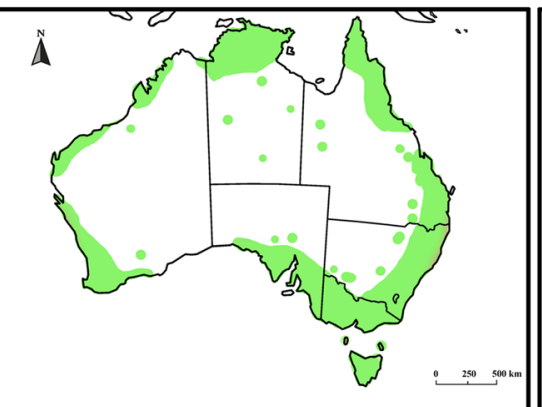

Rattus rattus

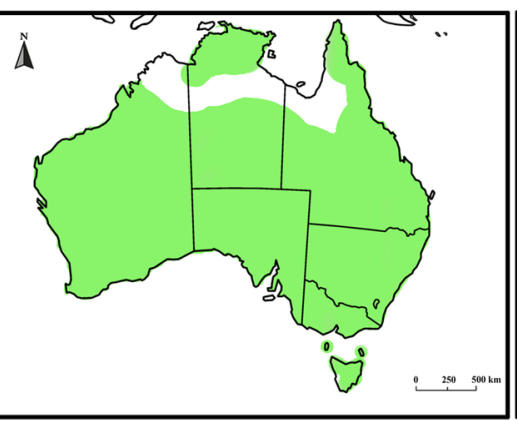

Mus musculus

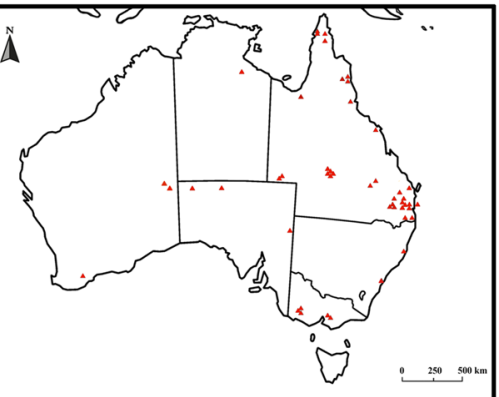

Rodents from which Polyplax spinulosa

was collected

Fig. 5 Distribution of introduced rodent species in Australia and locations of the rodents from which Polyplax spinulosa was collected in this study (adapted from https://ala.org.au)

cosmopolitan spiny rat louse, P. spinulosa, was introduced into Australia with its two hosts, the brown rat, $R$. norvegicus, and the black rat, $R$. rattus, by European explorers and colonizers [22, 36]. The earliest record of a European ship landing in Australia was in 1606 [37, 38], followed by British settlement in 1788 [39]. Thus, P. spinulosa would have had 200 to 400 years to expand and adapt to new hosts in Australia. The presence of $P$. spinulosa on endemic Rattus species in settled areas in Australia was briefly mentioned by Calaby and Murray [36]; however, there were no follow-up studies to ascertain whether these $P$. spinulosa lice were stragglers or established ectoparasites on the new host species. Only introduced $R$. norvegicus and $R$. rattus have been recognized as hosts of P. spinulosa in Australia [22]. 
Host switching by introduced sucking lice to endemic Australian rodents was reported only very recently. Wang et al. [10] showed for the first time that P. spinulosa had switched to, and established on, an endemic rodent, the long-haired rat, $R$. villosissimus. In the present study, we further investigated the host expansion by introduced sucking lice among the endemic rodents in Australia in more detail by examining 989 rodent specimens of 50 endemic species and four introduced species of murines deposited in seven museums across Australia. We also examined lice collected from two $R$. rattus specimens collected in Sabah (Borneo) and Sunshine Coast (Queensland), respectively. We found $P$. spinulosa on 63 individual rodents of three old endemic murine species and six new endemic murine species, in addition to the introduced host, $R$. rattus (Tables 1,2 ). We cannot exclude the presence of $P$. spinulosa on the golden-backed tree rat, Mesembriomys macrurus, Forrest's mouse, Leggadina forresti or the dusky rat, Rattus colletti, as stragglers as only a single $P$. spinulosa louse was found on each of these murine species (Tables 1 , 2). However, $P$. spinulosa was much more abundant on the other six old and new endemic rodent species: 12 to 706 adults of $P$. spinulosa specimens were found on each of these rodent species. In addition to the adult $P$. spinulosa, 9 to 73 Polyplax nymphs were also found on each of these rodent species. These nymphs cannot be identified to the species level but can be identified to the genus Polyplax (Fig. 4). Furthermore, P. spinulosa was found on multiple rodent individuals (4 to 17) from different locations collected over decades for all of the six rodent species except for Pesudomys occidentalis (Tables 1, 2). The 63 rodent specimens from which we collected P. spinulosa are mostly in Queensland but are distributed in all other Australian states except for Tasmania (Fig. 5). Our data indicate strongly that P. spinulosa has expanded its host range to at least six endemic rodent species in Australia in the time frame of 200 to 400 years. Outside Australia, P. spinulosa is known to parasitize eight endemic rat species in Eurasia where it originated; one of its sister species, $P$. serrata, parasitizes 10 mouse species (9 Apodemus spp. and Mus musculus) [7]. The extant host ranges of $P$. spinulosa and P. serrata indicate that these two species likely diverged $\sim 16$ MYA when rats and mice diverged [40-42]. The two genotypes indicated by our limited $r r n L$ sequence data also support an ancient origin of $P$. spinulosa in Eurasia. Therefore, the host range of P. spinulosa in Eurasia (i.e. 8 rat species) has taken millions of years to form. During this time period, P. spinulosa could have ample opportunities to switch and adapt to a much broader range of rodents (914 species in Eurasia) than in Australia (63 species) [43]. Apparently, the host expansion of $P$. spinulosa to at least six endemic murine species in Australia has occurred at a remarkably much faster pace than its host expansion in Eurasia. Furthermore, while P. spinulosa parasitizes only rats outside Australia, it expands to both endemic rats and an endemic mouse, Pseudomys occidentalis, in Australia.

\section{How did Polyplax spinulosa spread to its new hosts in Australia?}

Host switching by sucking lice usually requires physical contact between hosts, e.g. living in communal nests/ roosts, hosts breeding in close proximity, conspecific aggressive contact and during predator-prey contact [31]. Wang et al. [10] proposed that the wide distribution of the black rat, $R$. rattus, in Australia most likely facilitated the switch of $P$. spinulosa to the long-haired rat, $R$. villosissimus. This is also likely the case for the spread of $P$. spinulosa to the other endemic rodents revealed by the present study. The brown rat, $R$. norvegicus, and the Polynesian rat, $R$. exulans, are also hosts to $P$. spinulosa and are present in Australia as invasive species [22]. However, $R$. exulans is only found on a few offshore islands of Australia such as Adele Island and Norfolk Island but is absent from mainland Australia [12]. Rattus norvegicus inhabits primarily coastal urban areas of Australia, close to human populations [11]. Rattus rattus, however, is much more widely distributed than $R$. norvegicus and $R$. exulans. Rattus rattus can be found in coastal areas, in inland arid areas, on islands, in both human settlements or in areas with no human settlement in Australia (Fig. 5) [12]. Globally, $R$. rattus is one of the most successfully adapted invasive animal species and can be found on every continent except Antarctica [11]. The wide distribution of $R$. rattus would certainly create ample opportunities for it to have physical contacts with endemic rodents and for its parasites including $P$. spinulosa to transfer and adapt to new hosts. Overlapping distributions and physical contacts between endemic rodents could help $P$. spinulosa to expand its host range further once it had transferred from $R$. rattus to an endemic rodent species. All of the endemic rodent species on which we found $P$. spinulosa are abundant except for Pseudomys occidentalis and Mesembriomys macrurus, which are near threatened species on the ICUN Red List (https://www.iucnredlis t.org/). Five of these species (Leggadina forresti, R. fuscipes, $R$. lutreolus, Rattus tunneyi and $R$. villosissimus) have wide distributions in Australia, which would facilitate the host expansion of $P$. spinulosa. In particular, during population explosions, $R$. villosissimus can be found over an area of $130,000 \mathrm{~km}^{2}$ in high density (thus the common name, plague rat) [13]. The wide distribution and population explosions of $R$. villosissimus would certainly 
generate plenty of opportunities to either pick up or pass on $P$. spinulosa to other rodents. On the other hand, the rodent species on which we did not find any P. spinulosa are either those with localised distributions such as Rattus leucopus and Melomys capensis (only in the Cape York area), those near threated, vulnerable or endangered such as Pseudomys fieldi, Conilurus penicillatus, Leporillus conditor, Pseudomys fumeus (https://www.iucnr edlist.org/), or those with specialised habitats such as the water rat, Hydromys chrysogaster.

\section{Why did Polyplax spinulosa succeed in host expansions in Australia whereas Polyplax serrata and Hoplopleura pacifica failed?}

In addition to $P$. spinulosa, two other species of sucking lice, $H$. pacifica and $P$. serrata, have also been introduced into Australia with their commensal rodent hosts [22]. Like P. spinulosa, H. pacifica was introduced into Australia with the black rat, $R$. rattus, whereas $P$. serrata was introduced into Australia with the house mouse, $M$. musculus [22]. Outside Australia, H. pacifica has been recorded from six Rattus species including $R$. rattus, and P. serrata has been recorded from nine Eurasian Apodemus mouse species and M. musculus [7]. In stark contrast to $P$. spinulosa, neither $H$. pacifica nor $P$. serrata was found on any of the rodent specimens we examined in the present study (Tables 1, 2).

Why did $P$. spinulosa expand its host range successfully whereas $P$. serrata and $H$. pacifica failed? Sucking lice (Anoplura) are the most host-specific ectoparasites, are wingless, and feed only on host blood; this specialised life style, in general, limits their ability to transfer to, and establish on, new hosts $[2,7,44]$. However, the host specificity of sucking lice varies from species to species. Of the 532 species of sucking lice listed by Durden and Musser [3], 316 louse species are found on only a single host species, 92 louse species on two host species, 42 louse species on three host species, and 82 louse species including P. spinulosa, P. serrata and H. pacifica on four or more host species [7]. In the cases where one species of sucking louse parasitizes multiple host species, these hosts are almost always closely related, often in the same genus. Host species availability, however, is not the only factor that determines host specificity of sucking lice. The present study indicates that factors specific to each louse species also play a major role in determining the host specificity of sucking lice. These specific factors may pertain to the genetics or ecology of the louse species or its hosts, or both. The failure of $H$. pacifica to expand its host range in Australia is clearly due entirely to its own genetics or ecology because $H$. pacifica shares the same host, $R$. rattus, with $P$. spinulosa; host factors, thus, can be excluded in this case. The failure of $P$. serrata is more likely due to host factors. Polyplax serrata and P. spinulosa are closely related congeneric species with very similar morphology [45] and display similar host specificity outside Australia: $P$. serrata parasitizes 10 species of mice (9 Apodemus spp. and Mus musculus) whereas P. spinulosa parasitizes eight species of rats (seven Rattus spp. and Bandicota bengalensis) [7]. Polyplax serrata and P. spinulosa were introduced into Australia by $M$. musculus and R. rattus, respectively, through European exploration and colonization [46]. These two Polyplax species would have an approximately equal time frame (i.e. 200400 years) and an equal number of potential new hosts (i.e. 63 endemic mouse and rat species) on which to potentially expand. It is very likely that the ecology of $M$. musculus played a major role in the failure of $P$. serrata to expand its host range in Australia. In comparison to $R$. rattus, $M$. musculus is much more close to human settlements and less adaptable to utilizing environments without human settlements despite the fact that $M$. musculus is more widely distributed than $R$. rattus in Australia (Fig. 5) [13]. Thus, M. musculus would have significantly fewer opportunities for physical contacts with endemic rodents, which might have hampered the transfer of $P$. serrata to endemic rodents in Australia.

\section{Conclusions}

We have shown that $P$. spinulosa has expanded its host range to at least six endemic rat and mouse species in Australia in the time frame of 200 to 400 years since it was introduced, which is extremely rapid relative to its host expansion to eight native rat species in Eurasia in $\sim 16$ millions of years since it diverged from $P$. serrata. The host expansion of $P$. spinulosa is remarkable for a blood-sucking louse, and is in stark contrast to the absence of host expansion of $P$. serrata and $H$. pacifica in Australia. Comparison among these three introduced sucking lice indicates that both louse-specific factors and host-specific factors have contributed to host expansion. The successful host expansion of $P$. spinulosa can be attributed to both its genetics and ecology, plus the ecology of $R$. rattus, which carried $P$. spinulosa into Australia. The failure of $H$. pacifica to expand its host range is entirely due to its genetics or ecology because it shares the same host, $R$. rattus, with $P$. spinulosa. The failure of P. serrata, however, is very likely due to the ecology of its host, M. musculus, which is limited usually to human settlement areas and may not have sufficient opportunities to transfer $P$. serrata to endemic rodents. We expect further detailed comparative studies among these introduced sucking lice may pinpoint the exact factors of genetics or ecology that determine host specificity and host adaptation of sucking lice. 


\section{Supplementary information}

Supplementary information accompanies this paper at https://doi. org/10.1186/s13071-020-3957-y.

Additional file 1: Figure S1. Male Polyplax spinulosa collected from: (1) Rattus fuscipes; (2) Rattus lutreolus; (3) Rattus sordidus; (4) Rattus tunneyi; (5) Rattus villosissimus; (6) Rattus rattus; (7) Pseudomys occidentalis; and (8) Leggadina forresti (note: the shape of the abdomen can vary depending on the amount of distention from previous blood meals and the action of clearing chemicals prior to slide-mounting).

Additional file 2: Figure S2. Cleared female Polyplax spinulosa from different species of murine rodents: (1) Rattus colletti; (2) R. fuscipes; (3) R. lutreolus; (4) R. sordidus; (5) R. tunneyi; (6) R. villosissimus; (7) R. rattus; (8) Mesembriomys macrurus; and (9) Pseudomys occidentalis (note, the outline of an egg can be seen in 1) (note: the shape of the abdomen can vary depending on the amount of distention from previous blood meals and the action of clearing chemicals prior to slide-mounting).

Additional file 3: Figure S3. Thoracic sternal plates of Polyplax spinulosa collected from different species of Rattus: (1) ot from R. fuscipes; (2) o from $R$. fuscipes; (3) ot from $R$. lutreolus; (4) o from $R$. lutreolus; (5) ot from $R$. sordidus; (6) of from $R$. sordidus; (7) ot from R. tunneyi; (8) o from R. tunneyi; (9) of from $R$. villosissimus; (10) of from $R$. villosissimus; (11) ot from $R$. rattus; and (12) o from $R$. rattus.

Additional file 4: Figure S4. Sequence analysis of the mitochondrial cox1 gene of RLA, RVB, RS361 and Z65055 in comparison with EU162140. The four sites with nucleotide variation are indicated by red and green shading.

\section{Abbreviations}

MYA: million years ago; PCR: polymerase chain reaction; BLAST: Basic Local Alignment Search Tool; NJ consensus tree: neighbor-joining consensus tree; cox1: cytochrome c oxidase subunit 1; rrnL: $16 \mathrm{~S}$ ribosomal RNA; AGRF: Australian Genome Research Facilities; MV: Museums Victoria; QM: Queensland Museum; WAM: Western Australian Museum.

\section{Acknowledgements}

We thank Heather Janetzki (Queensland Museum), Karen Roberts, Katie Date and Kevin Rowe (Museums Victoria), Sandy Ingleby (Australian Museum), Kenny Travouillon and Rebecca Bray (Western Australian Museum), David Stemmer (South Australian Museum), Gavin Dally (Museum and Art Gallery of the Northern Territory), David Maynard and Judy Rainbird (Queen Victoria Museum and Art Gallery) for providing access to rodent specimens in museums. We thank Karin Koch (Queensland Museum), Nikolai Tatarnic (Western Australian Museum) and Ken Walker (Museums Victoria) for help with the registration of microscopic slides; and Fan Song (China Agricultural University), Guohua Liu (Hunan Agricultural University), Yalun Dong, Catherine Yule and Scott Burnett (University of the Sunshine Coast), Haylee Weaver (Australian Biological Resources Study), Rosie Booth and Ludovica Valenza (Australia Zoo Wildlife Hospital) and Konstans Wells (University of Swansea) for assisting with sample collection and/or identification. We thank Jan Štefka (The Biology Centre of the Czech Academy of Sciences) for providing the host information of a Polyplax spinulosa specimen (EU162140 in GenBank), Allison Morrison and Darren Morrow (University of the Sunshine Coast) for providing field work support. We thank Haylee Weaver for insightful comments on a previous draft of this manuscript.

\section{Authors' contributions}

WW, LD and RS designed this study. WW and RS collected louse specimens in museums. WW performed the DNA extraction, amplification, sequence analysis, phylogenetic analysis and morphological examination of the lice. WW and RS drafted the manuscript. WW, LD and RS edited and revised the manuscript. All authors read and approved the final manuscript.

\section{Funding}

This study was funded by the Australian Biological Resources Study (ABRS, RF217-51).

\section{Availability of data and materials}

Data supporting the conclusions of this article are included within the article. The gene sequences generated are available in the GenBank repository under the accession numbers MN193570-MN193577, MN427448 and MN434185.

The mounted microscopic slides of Polyplax spinulosa have been deposited in museums in Australia. The registration numbers of slides are: PHT-4 and PHT-5 (Museums Victoria), E102080 and E102081 (Western Australian Museum) and T246563-T246573 (Queensland Museum)

\section{Ethics approval and consent to participate}

Not applicable.

\section{Consent for publication}

Not applicable.

\section{Competing interests}

The authors declare that they have no competing interests.

\section{Author details}

${ }^{1}$ GeneCology Research Centre, School of Science and Engineering, University of the Sunshine Coast, Maroochydore, QLD 4556, Australia. ${ }^{2}$ Department of Biology, Georgia Southern University, Statesboro, GA 30458, USA.

Received: 24 October 2019 Accepted: 10 February 2020

Published online: 18 February 2020

\section{References}

1. Kim KC, Ludwig HW. The family classification of the Anoplura. Syst Entomol. 1978;3:249-84.

2. Light JE, Smith VS, Allen JM, Durden LA, Reed DL. Evolutionary history of mammalian sucking lice (Phthiraptera: Anoplura). BMC Evol Biol. 2010;10:292.

3. Durden LA, Musser GG. The mammalian hosts of the sucking lice (Anoplura) of the world: a host-parasite list. Bull Soc Vector Ecol. 1994:19:130-68.

4. Crystal MM. The mechanism of transmission of Haemobartonella muris (Mayer) of rats by the spined rat louse, Polyplax spinulosa (Burmeister). J Parasitol. 1958:44:603-6.

5. Badiaga S, Brouqui P. Human louse-transmitted infectious diseases. Clin Microbiol Infect. 2012;18:332-7.

6. Lehane MJ. The biology of blood-sucking in insects. New York: Cambridge University Press; 2005.

7. Durden LA, Musser GG. The sucking lice (Insecta, Anoplura) of the world: a taxonomic checklist with records of mammalian hosts and geographical distributions. Bull Am Mus Nat Hist. 1994;218:1-90.

8. Price RD, Hellenthal RA, Palma RL, Johnson KP, Clayton DH. The chewing lice: world checklist and biological overview, vol. 24. Chicago: Illinois Natural History Survey Special Publication; 2003.

9. Reed DL, Smith VS, Hammond SL, Rogers AR, Clayton DH. Genetic analysis of lice supports direct contact between modern and archaic humans. PLoS Biol. 2004;2:e340.

10. Wang W, Weaver HJ, Song F, Durden LA, Shao R. A new species of sucking louse Hoplopleura villosissima n. sp. (Psocodea: Phthiraptera: Hoplopleuridae) and a new host record of the spiny rat louse Polyplax spinulosa Burmeister (Psocodea: Phthiraptera: Polyplacidae) from the long-haired rat Rattus villosissimus Waite (Rodentia: Muridae) in Australia. Parasites Vectors. 1839:2018(11):476.

11. Musser GG, Carleton MD. Superfamily Muroidea. In: Wilson DE, Reeder DM, editors. Mammal species of the world: a taxonomic and geographic reference. 2nd ed. Baltimore: Johns Hopkins University Press; 2005. p. 894-1531.

12. Breed B, Ford F. Native mice and rats., Australian Natural History SeriesCollingwood: CSIRO Publishing; 2007.

13. Van Dyck S, Strahan R. The mammals of Australia. Sydney: New Holland Publishers; 2008.

14. Flannery T. Mammals of New Guinea. Sydney: Australian Museum/Reed New Holland; 1995. 
15. Ride WDL. Guide to the native mammals of Australia. Melbourne: Oxford Universty Press; 1970.

16. Robins JH, McLenachan PA, Phillips MJ, McComish BJ, Matisoo-Smith E, Ross HA. Evolutionary relationships and divergence times among the native rats of Australia. BMC Evol Biol. 2010;10:375.

17. Kim KC. A new species of Hoplopleura (Anoplura: Hoplopleuridae) from an Australian rat. Pac Insects. 1972;14:675-8.

18. Kuhn HJ, Ludwig HW. Sucking lice of the genus Hoplopleura (Anoplura: Insecta) from Australian Muridae. J Nat Hist. 1966;13:657-74.

19. Johnson PT. Some Anoplura of the oriental region. A study of Hoplopleura pacifca Ewing and Allies. J Med Entomol. 1972;9:219-27.

20. Weaver HJ, Barton PS. A new species of sucking louse (Phthiraptera: Anoplura) from Australia, and a key to the Australian species of Hoplopleura. Zootaxa. 2008;1679:55-62.

21. Weaver HJ. Three new species of the sucking louse genus Hoplopleura (Phthiraptera: Anoplura: Hoplopleuridae) from rodents (Mammalia: Rodentia: Muridae) in northern Australia. Zootaxa. 2017;4247:31-44.

22. Palma RL, Barker SC. Phthiraptera. In: Wells A, editor. Zoological catalogue of Australia, vol. 26., Psocoptera, Phthiraptera, ThysanopteraMelbourne: CSIRO Publishing: 1996. p. 81-247.

23. Palma RL. Slide-mounting of lice: a detailed description of the Canada balsam technique. N Z Entomol. 1978;6:432-6.

24. Kim KC, Pratt HD, Stojanovich CJ. The sucking lice of North America. An illustrated manual for identification. University Park: Pennsylvania State University Press; 1986.

25. Cruickshank RH, Johnson KP, Smith VS, Adams RJ, Clayton DH, Page RDM. Phylogenetic analysis of partial sequences of elongation factor 1 alpha identifies major groups of lice (Insecta: Phthiraptera). Mol Phylogenet Evol. 2001;19:202-15.

26. Johnson KP, Clayton $\mathrm{DH}$. Coevolutionary history of ecological replicates: comparing phylogenies of wing and body lice to columbiform hosts. In: Page RDM, editor. Tangled trees: phylogeny, cospeciation and coevolution. Chicago: University of Chicago Press; 2003. p. 262-86.

27. Simon CF, Frati F, Beckenbach AT, Crespi BJ, Liu H, Flook P. Evolution, weighting, and phylogenetic utility of mitochondrial gene sequences and a compilation of conserved polymerase chain reaction primers. Ann Entomol Soc Am. 1994;87:651-701.

28. Shao R, Zhu XQ, Barker SC, Herd K. Evolution of extensively fragmented mitochondrial genomes in the lice of humans. Genome Biol Evol. 2012:4:1088-101.

29. Zufall RA. Beyond simple homology searches: multiple sequence alignments and phylogenetic trees. Curr Protoc Essent Lab Tech. 2009:1:11-3.

30. Ferris GF. Contributions toward a monograph of the sucking lice, part IV. Stanford Univ Publ Univ Ser Biol Sci. 1923;2:183-270.

31. Durden LA, Musser GG. Sucking lice (Insecta, Anoplura) from indigenous Sulawesi rodents: a new species of Polyplax from a montane shrew rat, and new information about Polyplax wallacei and P. eropepli, vol. 3052. New York: American Museum Novitates; 1992.

32. Ferris GF. The sucking lice. Memoirs of the Pacific Coast Entomologica Society, vol. 1. San Francisco: The Pacific Coast Entomological Society; 1951
33. Johnson PT. The sucking lice (Anoplura) of Egypt: species infesting rodents. J Egypt Public Health Assoc. 1960;35:203-44.

34. Štefka J, Hypša V. Host specificity and genealogy of the louse Polyplax serrata on field mice, Apodemus species: a case of parasite duplication or colonisation? Int J Parasitol. 2008:38:731-41.

35. Yosida TH. Genetic aspects of polymorphic C-bands in the chromosomes of the black rat (Rattus rattus tanezumi) in Japan. Cytologia. 1979:44:265-74.

36. Calaby JH, Murray MD. Phthiraptera (Lice). In: Neumann ID, Came PB, Lawrence ES, Nielsen JP, Spradbery RW, Taylor MJ, et al., editors. The insects of Australia: a textbook for students and research workers. Melbourne: CSIRO: Melbourne University Press, Division of Entomology; 1991. p. 421-8.

37. Sheehan C. Voyages to the south land: Dutch explorations of the Queensland coast. Qld Hist J. 2010;21:72-87.

38. Mutch TD. The first discovery of Australia: with an account of the voyage of the "Duyfken" and the career of Captain Willem Jansz. J R Aust Hist Soc. 1942;28:2-55.

39. Gillen M, Browning Y, Flynn M. The founders of Australia: a biographical dictionary of the First Fleet. Sydney: Library of Australian History; 1989.

40. Douzery EJ, Delsuc F, Stanhope MJ, Huchon D. Local molecular clocks in three nuclear genes: divergence times for rodents and other mammals and incompatibility among fossil calibrations. J Mol Evol. 2003;57:201-13.

41. Smissen PJ, Rowe KC. Repeated biome transitions in the evolution of Australian rodents. Mol Phylogenet Evol. 2018;128:182-91.

42. Rowsey DM, Heaney LR, Jansa SA. Diversification rates of the "Old Endemic" murine rodents of Luzon Island, Philippines are inconsistent with incumbency effects and ecological opportunity. J Evol. 2018;72:1420-35.

43. Wilson DE, Reeder DM. Mammal species of the world: a taxonomic and geographic reference, vol. 1. 3rd ed. Baltimore: The Johns Hopkins University Press; 2005.

44. Kim KC. Blood-sucking lice (Anoplura) of small mammals: true parasites. In: Morand S, Krasnov BR, Poulin R, editors. Micromammals and macroparasites: from evolutionary ecology to management. New York: Springer; 2006. p. 141-60.

45. Sánchez-Montes S, Guzmán-Cornejo C, Ramírez-Corona F, León-Paniagua L. Anoplurans (Insecta: Psocodea: Anoplura) associated with rodents distributed in the Neotropical region of Mexico. Rev Mex Biodivers. 2016;87:427-35.

46. Gabriel SI, Stevens MI, Mathias Mda L, Searle JB. Of mice and "convicts": origin of the Australian house mouse, Mus musculus. PLoS ONE. 2011;6:e28622.

\section{Publisher's Note}

Springer Nature remains neutral with regard to jurisdictional claims in published maps and institutional affiliations.

Ready to submit your research? Choose BMC and benefit from

- fast, convenient online submission

- thorough peer review by experienced researchers in your field

- rapid publication on acceptance

- support for research data, including large and complex data types

- gold Open Access which fosters wider collaboration and increased citations

- maximum visibility for your research: over 100M website views per year

At BMC, research is always in progress.

Learn more biomedcentral.com/submissions 